\title{
Looking beyond the audiogram in ototoxicity associated with platinum-based chemotherapy
}

\author{
David M. Baguley ${ }^{1,2,3}$ (D) Pattarawadee Prayuenyong ${ }^{1,2,3,4}$ (D)
}

Received: 13 August 2019 / Accepted: 13 December 2019 / Published online: 21 December 2019

(c) The Author(s) 2019

\begin{abstract}
Introduction Ototoxicity associated with platinum-based chemotherapy is highly prevalent and can cause detrimental consequences among cancer survivors.

Discussion In this article, we highlight important aspects of the evaluation of ototoxicity with the aim to increase awareness of Oncologists in this regard. Standard pure tone audiometry alone is inadequate for this context. Comprehensive and consistent hearing tests should be implemented in a monitoring and surveillance program. High-frequency audiometry $(10-16 \mathrm{kHz})$ is a sensitive tool in the detection of ototoxic hearing loss at onset. In addition to threshold audiometry, measures of speech comprehension (both in quiet and in noise) can add useful information in the evaluation of hearing in real-life situations. Not only hearing loss, but also tinnitus and imbalance are common in patients who receive platinum-based chemotherapy, and can cause debilitating effects upon quality of life in this population. Moreover, self-report measures associated with cochlear and vestibular handicaps can provide valuable information regarding the impact of ototoxicity.

Conclusions It is vital to build awareness about the variety and impact of the symptoms of ototoxicity. Comprehensive evaluation of hearing status along with self-reported impact of the cochlear and vestibular handicap should be implemented in a monitoring and surveillance program for appropriate investigation and management.
\end{abstract}

Keywords Ototoxicity $\cdot$ Platinum-based chemotherapy $\cdot$ Hearing loss $\cdot$ Audiogram

\section{Introduction}

Platinum-based chemotherapies, such as cisplatin and carboplatin, are highly effective chemotherapeutic agents used for the treatment of a variety of life-threatening common cancers including testicular, gynaecologic, bladder, head and neck, and non-small cell lung cancer [1]. Despite potent efficacy against cancer, ototoxic effects are significantly problematic which limit usage and dosage [2]. Cisplatin has been

Pattarawadee Prayuenyong

msxpp4@nottingham.ac.uk

1 Hearing Sciences, Division of Clinical Neuroscience, School of Medicine, University of Nottingham, Nottingham, UK

2 NIHR Nottingham Biomedical Research Centre, Ropewalk House, 113 The Ropewalk, Nottingham, UK

3 Nottingham University Hospitals NHS Trust, Nottingham, UK

4 Department of Otorhinolaryngology Head and Neck Surgery, Faculty of Medicine, Prince of Songkla University, Songkhla, Thailand found to be the most ototoxic agent in the platinum-based chemotherapy group with associated hearing loss [3], tinnitus [4], and imbalance [5]. Carboplatin and oxaliplatin have been demonstrated to be less ototoxic [6], though audiovestibular issues still arise.

Ototoxicity refers to drug-related damage affecting the inner ear structures, specifically to the cochlea and the vestibular labyrinth, and their associated neural structures [3, 7]. Ototoxic effects can be characterized by cochlear dysfunction (such as hearing loss, tinnitus, or hyperacusis) or vestibular dysfunction (such as vertigo, dizziness, or imbalance) or both [7]. However, ototoxicity in the published literature usually refers to hearing disorders and both terms are used interchangeably [8].

Although hearing loss is not a fatal condition, it can have significant negative impacts on communication and healthrelated quality of life [9], and has been associated with dementia and cognitive impairment [10], poor mental health, and psychosocial functioning [11]. Approximately $70 \%$ of people with hearing loss had limited employment opportunities, failed to fulfill their potential at work, and felt isolated 
at work [12]. In children, hearing is substantially associated with speech and language development [13]; thus, hearing impairment can cause detrimental educational, vocational, and social consequences [14]. Additionally, it also has a hidden cost to society, such as reduced work productivity, if people are not offered appropriate interventions [15].

Early identification of ototoxicity might provide oncologists with an opportunity to adjust the chemotherapy regimen, either lowering the dosage or change to alternative drug, to reduce or prevent further hearing deterioration [16]. The primary aim of cancer treatment has been always to increase overall survival; however, quality of life has been increasingly documented as an important end point [17]. There is a lack of information on efficacy of ototoxicity monitoring and its cost-benefit ratio on treatment alteration [16]. There is limited information on the trade-off between longevity and quality-of-life cancer patient is willing to make [18]; therefore, the clinical decisions in oncology clinics are made on a case-by-case basis. In a study, Enoch et al. [19] suggested that hearing and balance were ranked in top three of the most valuable senses in a general adult population and participants preferred, on average, 6.8 years of perfect health over 10 years without hearing in a time trade-off exercise. This indicates that prolonged life with reduced hearing is of diminished value.

\section{Discussion}

Whilst there are many studies that report ototoxicity associated with platinum-based chemotherapy, the literature is characterized by small sample sizes, inadequate baseline measures, and non-standard reporting of audiometric measures. The prevalence of platinum-based ototoxicity in adults reported in the literature is approximately $50-80 \%[4,20]$ and $60-90 \%$ in children [21, 22]. A high inter-individual variability in incidence and severity of hearing loss can potentially be explained by differences in pharmacokinetics and pharmacodynamics of certain drugs including individual susceptibility factors such as genetics [23], and other co-morbid conditions such as renal diseases [24]. Some emerging clinical translational research indicates that prechemotherapy patient genotyping could help in predicting cisplatin-associated ototoxicity when deciding treatment regimens $[25,26]$. The severity of hearing loss associated with platinum-based chemotherapy within each individual seems to be dose-dependent and cumulative [27].

In this review, we argue that the prevalence of hearing loss associated with platinum-based chemotherapy can only be robustly determined by the consistent and appropriate use of measures of hearing in a strict pre, post, and longterm framework. Furthermore, we contend that the standard audiogram, a measure of the threshold of pure tone detection in quiet, is not a sufficient measure of real-world hearing. The use of extended high-frequency audiogram (HFA) $(10-16 \mathrm{kHz})$ facilitates early detection of ototoxic hearing loss at onset. Measures of speech comprehension, both in quiet and in noise, should also be utilized. Additionally, we discuss that treating ototoxicity as a synonym for hearing loss excludes the prevalent issues of tinnitus and/or imbalance found in this population. Finally, whilst self-report measures of auditory or vestibular handicap are imperfect tools, they should be brought to bear in studies of platinuminduced ototoxicity to determine the impact of ototoxicity.

\section{Monitoring ototoxic hearing loss}

Hearing loss caused by ototoxic medication has a relatively predictable pattern as it initially preferentially damages outer hair cells in the basal turn of the cochlea and then progresses to the apical turn [28]. Therefore, the classic characteristics of drug-induced hearing loss are bilaterally symmetrical sensorineural hearing loss that affects high frequencies, typically above $8 \mathrm{kHz}$ [1], which are key components of the discrimination of speech in background noise and music perception. Cochlear damage often progresses undetected until a substantial hearing communication problem becomes apparent suggesting hearing decline in the speech range frequencies.

Conventional pure tone audiometry (PTA) remains the mainstay for the identification and categorisation hearing impairment in many ototoxicity grading systems [29]. A PTA may be all the testing that patients undergoing chemotherapy can tolerate, and this may be especially true of the paediatric population [8]. In some younger children, otoacoustic emissions may present an opportunity to assess cochlear health in an ear and frequency-specific manner [8, $14,30]$. Audiological assessments for ototoxicity may differ from standard hearing evaluation in the priority of testing frequencies and sequence of testing [30]. High-frequency audiometry (HFA) is a more sensitive tool in the early identification of ototoxic changes than the standard PTA [31, 32]. However, HFA requires specific instrumentation and additional test time, and, in practice, a change in hearing higher than $8 \mathrm{kHz}$ generally does not impact the continuation of treatment regimen. Studies have revealed the ability to detect the early drug-induced cochlear damage through a limited behavioral test frequency range, called the sensitive range of ototoxicity (SRO) [33]. The SRO is a pure tone screening procedure in which a one-octave individualized range of frequencies at the high-frequency limit of hearing is monitored. The SRO is defined as the highest frequency with a threshold $\leq 100 \mathrm{~dB}$ followed by six lower consecutive frequencies in 1/6th-octave steps; thus, it is unique for each individual's audiometric configuration. Testing these seven frequencies identifies approximately $90 \%$ of initial ototoxic 
hearing shifts [34]. The SRO procedure is both sensitive and time-efficient technique. Identifying the SRO is relatively quick while maintaining the sensitivity compared with PTA and HFA, and can be assessed using an extended high-frequency audiometer [33].

Clinically, ototoxicity is diagnosed by comparing functional status before and after the administration of ototoxic drugs; hence, baseline evaluation is essential. This prevents inaccurate diagnosis of iatrogenic ototoxic hearing loss actually caused by previous hearing impairment prior to chemotherapy treatment such as presbycusis or noise-induced hearing loss. This is particularly important in adult population as those conditions share similar audiometric results to those caused by ototoxic medications. Obtaining pre- and post-treatment hearing assessments also support basic and clinical research on drugs or interventions that can neutralize ototoxicity while not interfering with the efficacy of the antineoplastic capabilities of chemotherapy. Pre-existing hearing status in combination with cisplatin cumulative dose can be useful in the prediction of the degree of ototoxic hearing loss [35]. A tool to help relatively precise predictions regarding the potential reduction in hearing in advance of chemotherapy treatment would be valuable for pretreatment counselling and oncology treatment planning.

Drug-induced hearing loss is generally irreversible and occurs in a dose-related and cumulative fashion $[35,36]$. Consequently, a regular monitoring program is crucial for the early detection of ototoxicity which provides useful information to minimize irreversible hearing loss as well as timely interventions. Hearing impairment after administration of platinum-based compounds can also be progressive for years after discontinuation of medication [14], which means that hearing loss may not only evident in patients who sustained ototoxicity during treatment. In addition, recent evidence shows that platinum is retained indefinitely in patients treated with cisplatin [37]. Thus, long-term surveillance is necessary because of the potential for progressive or delayed-onset hearing loss. American Speech-LanguageHearing Association (ASHA) recommends evaluations to be done in 1 and 3 months following discontinuation of ototoxic therapy [38]. Recent evidence-based guidance on ototoxicity monitoring in adolescents and young adults proposed ongoing 5 yearly audiometry [8].

The use of different criteria and grading systems for ototoxicity has made the analysis of published data challenging to interpret and has partly contributed to the variability in reporting the prevalence of drug-induced hearing loss throughout the literature [39]. Hence, uniformity of classification systems is essential to compare the results in both clinical practice and trial settings. A recent review article summarized numerous classification systems developed and used to classify cochleotoxicity using different audiometric criteria [29]. For example, the Common Terminology
Criteria for Adverse Events (CTCAE) are widely accepted among the oncology research community as the standard grading scale for adverse events in clinical trials. Audiometric results are graded (1-4) according to the threshold change, number of frequencies affected, and indication for intervention. However, the CTCAE version 4 (2010) and 5 (2017) do not fully encapsulate the functional difference in difficulties experienced between a change in threshold from different baselines [29].

All of the available information emphasizes the importance of coherent and proper hearing measurement before, during, and after chemotherapy to determine the incidence and prevalence of hearing impairment associated with platinum-based chemotherapy. However, such monitoring programs are not routinely implemented [40] despite the existence of clinical guidelines $[16,38]$ and recommendations for ototoxicity surveillance [8].

\section{Threshold measures of hearing are insufficient: discrimination measures are needed}

A complaint expressed by people with hearing impairment is of hearing difficulties in noisy environments [41], though, sometimes, hearing loss is not detected with routine hearing testing [42]. PTA, measuring tone detection threshold of varying frequency in quiet condition, is a poor indicator of speech recognition in noise ability, so it may not reflect dynamic real-world hearing status nor predict the handicap produced by hearing loss [43].

Speech discrimination abilities may be affected as well as detection of hearing thresholds after receiving platinumbased chemotherapy [44]. Hearing in noise testing is not commonly utilised in ototoxicity monitoring programs [16, 38], so challenges in hearing discrimination and intelligibility of speech are not routinely assessed. We propose that the evaluation of hearing perception in the presence of noise in addition to the hearing in quiet conditions will add valuable information in comprehensive hearing evaluation and help with hearing rehabilitation plan and, therefore, should be implemented in clinical practice. Examples of clinicallyfeasible speech-in-noise tests that are quick to administer are the Words-In-Noise (WIN) test and Quick Speech-In-Noise Test (QuickSIN) [45].

\section{Tinnitus}

Tinnitus is a subjective perception of sound, for example, hissing, whistling or buzzing, without an external source [46], and which can lead to significant negative impacts on psychological status and quality of life [47]. Tinnitus is more prevalent in patients with hearing loss compared to normal hearing populations [48, 49]. Regarding ototoxicity, in a series of adult patients treated with cisplatin, 
$59 \%$ experienced tinnitus, whereas $18 \%$ had hearing loss only and $23 \%$ had both symptoms [50]. Frisina et al. [4] reported approximately $40 \%$ of testicular cancer survivors who received cisplatin complained of tinnitus which was significantly correlated with reduced hearing. On the other hand, Arora et al. [51] revealed 6 out of 57 (10.5\%) patients had tinnitus irrespective of the dose of cisplatin and none of them had complained of subjective hearing loss. A recent study evaluated long-term ototoxicity in pediatric patients received platinum-based chemotherapy and/or radiotherapy reported that $66.7 \%$ of patients reported tinnitus, although they may have normal hearing detected by standard audiometry [52]. The prevalence of tinnitus associated with platinum-induced ototoxicity is unclear in the literature because of the scant research in this area, but it is likely to be underreported and underappreciated [21].

\section{Vestibular symptoms}

Vestibular effects associated with platinum-based chemotherapy are less frequently described in the literature compared to auditory symptoms, but can be debilitating [5]. Vestibular dysfunction can trigger a deterioration of quality of life including physical impairment that interfere with driving, riding a bicycle, and other activities involving good balance, as well as psychological symptoms [53]. More than 50\% of subjects with dizziness report reduced efficiency at work, $27 \%$ changed their jobs, $21 \%$ gave up work, and patients report other considerable impacts on personal and social life [54].

Ototoxic medications are generally administered systemically and, therefore, affect both ears simultaneously. Bilateral symmetrical gradual vestibular loss usually results in insidious disequilibrium, postural imbalance, or oscillopsia [55]. A compensated vestibular loss may not be recognized until the patient loses other cues from vision and somatosensory inputs such as when walking in the dark or when concomitant peripheral neuropathy is developed [5]. Moreover, there are multiple factors such as the general deconditioning of cancer patients that can make the clinical identification of vestibulotoxicity more complicated. Most patients are unlikely to have intense symptoms of imbalance; hence, subtle or suspicious symptoms of vestibular impairment should be recorded and/or undergo further investigations, for example, vertigo, dizziness, double vision, ataxia, and light-headedness $[55,56]$. Vestibulotoxicity associated with platinumbased chemotherapy seems to be underinvestigated and underestimated [5]. Clinicians should be vigilant to the presenting symptoms of vestibular impairment in this patient population.

\section{Measurement of impact}

Although testing can detect hearing loss at an early stage, the impact upon daily activities and quality of life as assessed by self-report is also important. One common limitation of the available cochleotoxicity grading systems is a lack of indication of significant clinical change of hearing linked to reduced communication function and quality of life [29]. Similar audiological characteristics in different individuals may demonstrate varying degrees of communication difficulties [57]. Self-reports of hearing difficulty generally have a higher prevalence than test measures in population studies [43], and patients may have a substantially greater hearing handicap and disability than would be expected from the results of the audiogram [44].

Self-report measures of communication difficulty should complement audiometric examinations in monitoring protocol to guide treatment plans and hearing rehabilitation as well as to gain a better understanding of the incidence and burden of ototoxicity. An example of patient-reported tools is the Scale of ChemotherapyInduced Neurotoxicity (SCIN) [58]. Patients answer in four categories 'not at all', 'a little', 'quite a bit', and 'very much' to the questions: 'Have you suffered from reduced hearing?' and 'Have you suffered from ringing in your ears?'. The impact of tinnitus and dizziness on quality of life can be evaluated using the Tinnitus Handicap Inventory (THI) [59], and the Dizziness Handicap Inventory (DHI) [60], respectively. The use of questionnaires in the paediatric population is not presently supported by validated instruments.

\section{Conclusions}

Ototoxicity associated with platinum-based chemotherapy is a salient issue. It should be a priority to build awareness among patients and healthcare providers about the significance and variety of symptoms of ototoxicity such as reduced hearing, tinnitus, and imbalance. Comprehensive and robust baseline hearing tests within a monitoring and surveillance program should be scheduled to assess prevalence of hearing loss associated with platinum-based chemotherapy. Measures of speech-in-noise complement speech discrimination testing in quiet conditions and can add helpful information in the evaluation of real-life hearing abilities. Furthermore, the potential impact of cochlear and vestibular handicap caused by ototoxicity should be assessed by selfreport measures.

Acknowledgements David Baguley is supported by the UK National Institute of Health Research (NIHR), but his views herein are his own, 
and do not reflect those of the NIHR nor the Department of Health and Social Care

\section{Compliance with ethical standards}

Conflict of interest The authors have no potential conflicts of interest to disclose.

Open Access This article is licensed under a Creative Commons Attribution 4.0 International License, which permits use, sharing, adaptation, distribution and reproduction in any medium or format, as long as you give appropriate credit to the original author(s) and the source, provide a link to the Creative Commons licence, and indicate if changes were made. The images or other third party material in this article are included in the article's Creative Commons licence, unless indicated otherwise in a credit line to the material. If material is not included in the article's Creative Commons licence and your intended use is not permitted by statutory regulation or exceeds the permitted use, you will need to obtain permission directly from the copyright holder. To view a copy of this licence, visit http://creativecommons.org/licenses/by/4.0/.

\section{References}

1. Kros CJ, Steyger PS (2018) Aminoglycoside- and cisplatininduced ototoxicity: mechanisms and otoprotective strategies. Cold Spring Harbor Perspect Med. https://doi.org/10.1101/cshpe rspect.a033548

2. Trendowski MR, El Charif O, Dinh PC Jr et al (2019) Genetic and modifiable risk factors contributing to cisplatin-induced toxicities. Clin Cancer Res 25(4):1147-1155. https://doi.org/10.1158/10780432.ccr-18-2244

3. Sheth S, Mukherjea D, Rybak LP et al (2017) Mechanisms of cisplatin-induced ototoxicity and otoprotection. Front Cell Neurosci 11:338. https://doi.org/10.3389/fncel.2017.00338

4. Frisina RD, Wheeler HE, Fossa SD et al (2016) Comprehensive audiometric analysis of hearing impairment and tinnitus after cisplatin-based chemotherapy in survivors of adult-onset cancer. J Clin Oncol 34(23):2712-2720. https://doi.org/10.1200/ JCO.2016.66.8822

5. Prayuenyong P, Taylor JA, Pearson SE et al (2018) Vestibulotoxicity associated with platinum-based chemotherapy in survivors of cancer: a scoping review. Front Oncol 8:363. https://doi. org/10.3389/fonc.2018.00363

6. Ding D, Allman BL, Salvi R (2012) Review: ototoxic characteristics of platinum antitumor drugs. Anat Record (Hoboken, NJ: 2007) 295(11):1851-1867. https://doi.org/10.1002/ar.22577

7. Cianfrone G, Pentangelo D, Cianfrone F et al (2011) Pharmacological drugs inducing ototoxicity, vestibular symptoms and tinnitus: a reasoned and updated guide. Eur Rev Med Pharmacol Sci 15(6):601-636

8. Clemens E, van den Heuvel-Eibrink MM, Mulder RL et al (2019) Recommendations for ototoxicity surveillance for childhood, adolescent, and young adult cancer survivors: a report from the international late effects of childhood cancer guideline harmonization group in collaboration with the pancare consortium. Lancet Oncol 20(1):e29-e41. https://doi.org/10.1016/s1470-2045(18)30858-1

9. Punch JL, Hitt R, Smith SW (2019) Hearing loss and quality of life. J Commun Disord 78:33-45. https://doi.org/10.1016/j.jcomd is. 2019.01 .001

10. Thomson RS, Auduong P, Miller AT et al (2017) Hearing loss as a risk factor for dementia: a systematic review. Laryng Investig Otolaryngol 2(2):69-79. https://doi.org/10.1002/lio2.65
11. Lawrence BJ, Jayakody DMP, Bennett RJ et al (2019) Hearing loss and depression in older adults: a systematic review and metaanalysis. The Gerontologist. https://doi.org/10.1093/geront/gnz00 9

12. Archbold S, Lamb B, O'Neill C et al (2018) The real cost of adult hearing loss: reducing its impact by increasing access to the latest hearing technologies. The Ear Foundation, Nottingham

13. Dimitrov L, Jan A, Bhimji SS (2019) Pediatric hearing loss In Statpearls. StatPearls Publishing LLC., Florida

14. Knight KR, Kraemer DF, Neuwelt EA (2005) Ototoxicity in children receiving platinum chemotherapy: underestimating a commonly occurring toxicity that may influence academic and social development. J Clin Oncol 23(34):8588-8596. https://doi. org/10.1200/jco.2004.00.5355

15. Graydon K, Waterworth C, Miller H et al (2019) Global burden of hearing impairment and ear disease. Journal Laryngol Otol 133(1):18-25. https://doi.org/10.1017/s0022215118001275

16. American Academy of Audiology (2009) American academy of audiology position statement and guidelines: Ototoxicity monitoring. American Academy of Audiology. https://audio logy-web.s3.amazonaws.com/migrated/OtoMonGuideline s.pdf_539974c40999c1.58842217.pdf

17. Khan FA, Akhtar SS, Sheikh MK (2005) Cancer treatmentobjectives and quality of life issues. Malays J Med Sci 12(1):3-5

18. Shrestha A, Martin C, Burton M et al (2019) Quality of life versus length of life considerations in cancer patients: a systematic literature review. Psychooncology 28(7):1367-1380. https://doi. org/10.1002/pon.5054

19. Enoch J, McDonald L, Jones L et al (2019) Evaluating whether sight is the most valued sense. JAMA Ophthalmol. https://doi. org/10.1001/jamaophthalmol.2019.3537

20. Skalleberg J, Solheim O, Fossa SD et al (2017) Long-term ototoxicity in women after cisplatin treatment for ovarian germ cell cancer. Gynecol Oncol 145(1):148-153. https://doi. org/10.1016/j.ygyno.2017.02.006

21. van As JW, van den Berg H, van Dalen EC (2016) Platinuminduced hearing loss after treatment for childhood cancer. Cochrane Database Syst Rev. https://doi.org/10.1002/14651 858.CD010181.pub2

22. Bass JK, Huang J, Onar-Thomas A et al (2014) Concordance between the chang and the international society of pediatric oncology (siop) ototoxicity grading scales in patients treated with cisplatin for medulloblastoma. Pediatr Blood Cancer 61(4):601-605. https://doi.org/10.1002/pbc.24830

23. Tserga E, Nandwani T, Edvall NK et al (2019) The genetic vulnerability to cisplatin ototoxicity: a systematic review. Sci Rep 9(1):3455. https://doi.org/10.1038/s41598-019-40138-Z

24. Landier W, Knight K, Wong FL et al (2014) Ototoxicity in children with high-risk neuroblastoma: prevalence, risk factors, and concordance of grading scales-a report from the children's oncology group. J Clin Oncol 32(6):527-534. https://doi. org/10.1200/jco.2013.51.2038

25. Wheeler HE, Gamazon ER, Frisina RD et al (2017) Variants in wfs 1 and other mendelian deafness genes are associated with cisplatin-associated ototoxicity. Clin Cancer Res 23(13):33253333. https://doi.org/10.1158/1078-0432.Ccr-16-2809

26. Travis LB, Fossa SD, Sesso HD et al (2014) Chemotherapyinduced peripheral neurotoxicity and ototoxicity: new paradigms for translational genomics. J Natl Cancer Inst. https:// doi.org/10.1093/jnci/dju044

27. Esfahani Monfared Z, Khosravi A, Safavi Naini A, et al (2017) Analysis of cisplatin-induced ototoxicity risk factors in Iranian patients with solid tumors: a cohort, prospective and single institute study. APJCP 18(3):753-758. 10.22034/ apjcp.2017.18.3.753. 
28. Gauvin DV, Yoder J, Zimmermann ZJ et al (2018) Ototoxicity: the radical drum beat and rhythm of cochlear hair cell life and death. Int J Toxicol 37(3):195-206. https://doi.org/10.1177/1091581818 761128

29. Crundwell G, Gomersall P, Baguley DM (2016) Ototoxicity (cochleotoxicity) classifications: a review. Int J Audiol 55(2):6574. https://doi.org/10.3109/14992027.2015.1094188

30. Brooks B, Knight K (2017) Ototoxicity monitoring in children treated with platinum chemotherapy. Int J Audiol 57:1-7. https:// doi.org/10.1080/14992027.2017.1355570

31. Fausti SA, Larson VD, Noffsinger D et al (1994) High-frequency audiometric monitoring strategies for early detection of ototoxicity. Ear Hear 15(3):232-239

32. Abujamra AL, Escosteguy JR, Dall'Igna C et al (2013) The use of high-frequency audiometry increases the diagnosis of asymptomatic hearing loss in pediatric patients treated with cisplatinbased chemotherapy. Pediatr Blood Cancer 60(3):474-478. https ://doi.org/10.1002/pbc.24236

33. Fausti SA, Helt WJ, Phillips DS et al (2003) Early detection of ototoxicity using 1/6th-octave steps. J Am Acad Audiol 14(8):444-450

34. Fausti SA, Henry JA, Helt WJ et al (1999) An individualized, sensitive frequency range for early detection of ototoxicity. Ear Hear 20(6):497-505

35. Dille MF, Wilmington D, McMillan GP et al (2012) Development and validation of a cisplatin dose-ototoxicity model. J Am Acad Audiol 23(7):510-521. https://doi.org/10.3766/jaaa.23.7.3

36. Yancey A, Harris MS, Egbelakin A et al (2012) Risk factors for cisplatin-associated ototoxicity in pediatric oncology patients. Pediatr Blood Cancer 59(1):144-148. https://doi.org/10.1002/ pbc. 24138

37. Breglio AM, Rusheen AE, Shide ED et al (2017) Cisplatin is retained in the cochlea indefinitely following chemotherapy. Nat Commun 8(1):1654. https://doi.org/10.1038/s41467-017-01837-1

38. Association AS-L-H (1994) Guidelines for the audiologic management of individuals receiving cochleotoxic drug therapy. ASHA 36(Suppl 12):11-19

39. Neuwelt EA, Brock P (2010) Critical need for international consensus on ototoxicity assessment criteria. J Clin Oncol 28(10):1630-1632. https://doi.org/10.1200/jco.2009.26.7872

40. Maru D, Malky GA (2018) Current practice of ototoxicity management across the united kingdom (UK). Int J Audiol 57(sup4):S76-s88. https://doi.org/10.1080/14992027.2018.14604 95

41. Vermiglio AJ, Soli SD, Freed DJ et al (2012) The relationship between high-frequency pure-tone hearing loss, hearing in noise test (hint) thresholds, and the articulation index. J Am Acad Audiol 23(10):779-788. https://doi.org/10.3766/jaaa.23.10.4

42. Kenyon EL, Leidenheim SE, Zwillenberg S (1998) Speech discrimination in the sensorineural hearing loss patient: How is it affected by background noise? Mil Med 163(9):647-650

43. Moore DR, Edmondson-Jones M, Dawes P et al (2014) Relation between speech-in-noise threshold, hearing loss and cognition from 40-69 years of age. PLoS ONE 9(9):e107720. https://doi. org/10.1371/journal.pone.0107720

44. Einarsson EJ, Petersen H, Wiebe T et al (2010) Long term hearing degeneration after platinum-based chemotherapy in childhood. Int J Audiol 49(10):765-771. https://doi.org/10.3109/14992 027.2010.485595

45. Portnuff C, Bell B (2019) Effective use of speech-in-noise testing in the clinic. Hear J 72(5):40-43. https://doi.org/10.1097/01. HJ.0000559502.51932.b1
46. Baguley D, McFerran D, Hall D (2013) Tinnitus. Lancet (London, England) 382(9904):1600-1607. https://doi.org/10.1016/s0140 -6736(13)60142-7

47. Manchaiah V, Beukes EW, Granberg S et al (2018) Problems and life effects experienced by tinnitus research study volunteers: An exploratory study using the ICF classification. J Am Acad Audiol 29(10):936-947. https://doi.org/10.3766/jaaa.17094

48. Tan CM, Lecluyse W, McFerran D et al (2013) Tinnitus and patterns of hearing loss. J Assoc Res Otolaryngol JARO 14(2):275282. https://doi.org/10.1007/s10162-013-0371-6

49. Aazh H, Salvi R (2018) The relationship between severity of hearing loss and subjective tinnitus loudness among patients seen in a specialist tinnitus and hyperacusis therapy clinic in UK. J Am Acad Audiol. https://doi.org/10.3766/jaaa.17144

50. Bokemeyer C, Berger CC, Hartmann JT et al (1998) Analysis of risk factors for cisplatin-induced ototoxicity in patients with testicular cancer. Br J Cancer 77(8):1355-1362

51. Arora R, Thakur JS, Azad RK et al (2009) Cisplatin-based chemotherapy: add high-frequency audiometry in the regimen. Indian $\mathrm{J}$ Cancer 46(4):311-317. https://doi.org/10.4103/0019-509x.55551

52. Waissbluth S, Chuang A, Del Valle A et al (2018) Long term platinum-induced ototoxicity in pediatric patients. Int J Pediatr Otorhinolaryngol 107:75-79. https://doi.org/10.1016/j.ijpor 1.2018.01.028

53. Sun DQ, Ward BK, Semenov YR et al (2014) Bilateral vestibular deficiency: quality of life and economic implications. JAMA Otolaryngol Head Neck Surg 140(6):527-534. https://doi. org/10.1001/jamaoto.2014.490

54. Bronstein AM, Golding JF, Gresty MA et al (2010) The social impact of dizziness in london and siena. J Neurol 257(2):183-190. https://doi.org/10.1007/s00415-009-5287-z

55. Lucieer F, Vonk P, Guinand N et al (2016) Bilateral vestibular hypofunction: insights in etiologies, clinical subtypes, and diagnostics. Front Neurol 7:26. https://doi.org/10.3389/fneur .2016 .00026

56. van de Berg R, van Tilburg M, Kingma H (2015) Bilateral vestibular hypofunction: challenges in establishing the diagnosis in adults. ORL J Oto-Rhino-Laryngol Relat Spec 77(4):197-218. https://doi.org/10.1159/000433549

57. Hallberg LR, Hallberg U, Kramer SE (2008) Self-reported hearing difficulties, communication strategies and psychological general well-being (quality of life) in patients with acquired hearing impairment. Disabil Rehabil 30(3):203-212. https://doi. org/10.1080/09638280701228073

58. Oldenburg J, Fossa SD, Dahl AA (2006) Scale for chemotherapyinduced long-term neurotoxicity (scin): psychometrics, validation, and findings in a large sample of testicular cancer survivors. Quality of life Res 15(5):791-800. https://doi.org/10.1007/s1113 6-005-5370-6

59. Newman CW, Jacobson GP, Spitzer JB (1996) Development of the tinnitus handicap inventory. Arch Otolaryngol Head Neck Surg 122(2):143-148

60. Jacobson GP, Newman CW (1990) The development of the dizziness handicap inventory. Arch Otolaryngol Head Neck Surg 116(4):424-427

Publisher's Note Springer Nature remains neutral with regard to jurisdictional claims in published maps and institutional affiliations. 